\title{
Performance Limit Projection of Germanane Field-Effect Transistors
}

\author{
AbdulAziz AlMutairi, Yiju Zhao, Demin Yin, and Youngki Yoon, Member, IEEE
}

\begin{abstract}
Here we explore the performance limit of monolayer germanane (GeH) field-effect transistors (FETs). We first plotted an electronic band structure of $\mathrm{GeH}$ using density functional theory (DFT) and then tight-binding parameters were extracted. Device characteristics of GeH FETs are investigated using rigorous self-consistent atomistic quantum transport simulations within tight-binding approximations. Our simulation results indicate that GeH FETs can exhibit exceptional on-state device characteristics such as high $I_{o n}(>2 \mathrm{~mA} / \mu \mathrm{m})$ and large $g_{m}(\sim 7$ $\mathrm{mS} / \mu \mathrm{m})$ with $V_{D D}=0.5 \mathrm{~V}$ due to the very light effective mass of GeH $\left(0.07 m_{0}\right)$, while maintaining excellent switching characteristics (SS $\sim 64 \mathrm{mV} / \mathrm{dec}$ ). We have also performed a scaling study by varying the channel length, and it turned out that GeH FET can be scaled down to $\sim 14 \mathrm{~nm}$ channel without facing significant short channel effects but it may suffer from large leakage current at the channel length shorter than $10 \mathrm{~nm}$. Finally, we have benchmarked GeH FET against $\mathrm{MoS}_{2}$ counterpart, exhibiting better suitability of $\mathrm{GeH}$ device for high-performance applications compared to $\mathrm{MoS}_{2}$ transistors.
\end{abstract}

Index Terms - Germanane, Field-Effect Transistor, Quantum Transport, Non-Equilibrium Green's Function, Device Simulation

\section{INTRODUCTION}

$\mathrm{N}$ anoelectronics based on two-dimensional (2D) material has shown great potential in the last decade. 2D materials like graphene, transition metal dichalcogenides (TMDs) and black phosphorus have proven their utility in various applications such as field-effect transistors (FETs) [1], [2], memory devices [3], and optoelectronics applications [4], [5]. Due to its high carrier mobility and saturation velocity [6], [7], recent graphene research has been mainly focused on high-frequency applications [6]. On the other hand, TMDs like molybdenum disulfide $\left(\mathrm{MoS}_{2}\right)$ have been widely explored for low-power switching applications in light of the large band gap and exceptional electrostatic integrity [1].

Recently, germanane $(\mathrm{GeH})$ has emerged as a new family of $2 \mathrm{D}$ materials, which is a single layer of germanium $(\mathrm{Ge})$ with hydrogen $(\mathrm{H})$ atoms attached in the out-of-plane direction [8]. It can be synthesized through topotactic deintercalation of $\mathrm{CaGe}_{2}$ [8], and first-principle studies showed its very light effective mass and ultra-high carrier mobility $(>18,000$ $\mathrm{cm}^{2} / \mathrm{V} \cdot \mathrm{s}$ ) [8], [9]. However, FETs based on $\mathrm{GeH}$ as an active

The first two authors contributed equally to this work. This work was supported in part by NSERC Discovery Grant (RGPIN-05920-2014) and in part by NSERC Strategic Project Grant (STPGP 478974-15). Computing resources were provided by SHARCNET through Compute Canada. Demin Yin acknowledges the financial support by WIN Nanofellowship.

The authors are with Department of Electrical and Computer Engineering \& Waterloo Institute for Nanotechnology (WIN) at the University of Waterloo, Waterloo, ON, Canada N2L 3G1 (Email: youngki.yoon@uwaterloo.ca). (a) $)_{3}$

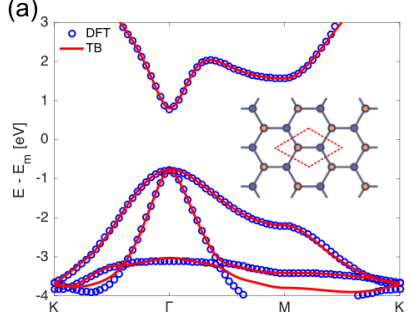

(b)

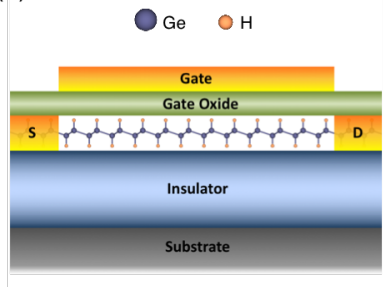

Fig. 1 (a) Electronic band structure of germanane $(\mathrm{GeH})$ based on density functional theory (blue circles), which is fitted with tight-binding parameters (solid red lines). (Inset) the top view of germanane lattice with the unit cell represented by red dotted lines. (b) Device structure for the simulated $\mathrm{GeH}$ field-effect transistor (GeH FET).

channel material have rarely explored although it has great potential for future electronic devices toward various applications. The performance of GeH FET was estimated previously by semi-classical model [10], but such a simple approach can be significantly limited in predicting the detailed characteristics of germanane device, where quantum-mechanical treatment will be critical to discuss tunneling and scaling. Therefore, in this work, we investigate the performance limit of $\mathrm{GeH}$ FET using rigorous self-consistent atomistic quantum transport simulations. Our simulation results exhibit superior on-state characteristics of GeH FET with excellent switching behaviors. However, due to the very light effective mass, the scaling of GeH FET can be significantly limited as it suffers from large leakage current. We have also benchmarked $\mathrm{GeH}$ FET against $\mathrm{MoS}_{2}$ counterpart, which indicates that GeH FET has clear benefits for high-performance applications compared to a similar device based on $\mathrm{MoS}_{2}$.

\section{Simulation MethodS}

Density functional theory (DFT) calculation [11] was utilized to obtain the band structure of $\mathrm{GeH}$. Generalized gradient approximations (GGA) exchange correlation function using PBE parameterization was employed for the DFT calculation. For a geometry optimization, conjugate-gradients (CG) was used with maximum force tolerance of $10^{-3} \mathrm{eV} / \AA$ and energy tolerance of $10^{-4} \mathrm{eV}$. The unit cell of germanane contains two germanium atoms and two hydrogen atoms as shown in the inset of Fig. 1 (a). The Brillouin zone sampling was done using Monkhorst-Pack approach with a $50 \times 50 \times 1$ mesh. The calculated band structure is shown in Fig. 1(a), exhibiting isotropic effective masses around the $\Gamma$ point. The electron effective mass is $0.07 m_{0}$ and those of heavy hole and light hole are $0.50 m_{0}$ and $0.07 m_{0}$, receptively, where $m_{0}$ is free electron mass, showing good agreements with the reported values in literature [8], [9]. We have corrected the underestimated band gap $\left(E_{g}\right)$ from our DFT calculation to 1.56 
(a)

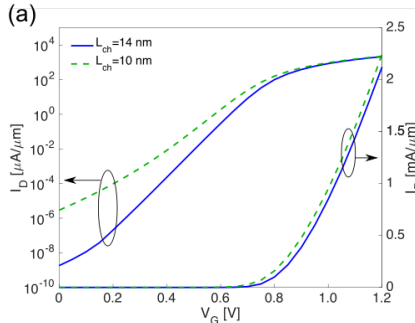

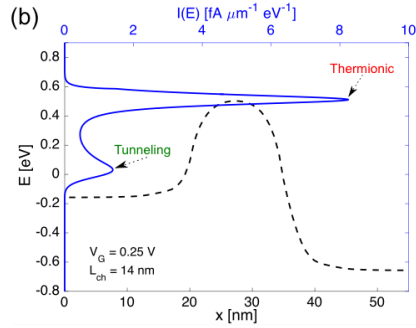

Fig. 2 (a) Transfer characteristics of monolayer GeH FET with two different channel lengths $\left(L_{c h}=10\right.$ and $\left.14 \mathrm{~nm}\right)$ at $V_{D}=0.5 \mathrm{~V}$. (b) Energy-resolved current spectrum (blue solid line; top axis) and conduction band $\left(E_{c}\right)$ profile along the device (black dashed line; bottom axis) for 14-nm channel at $V_{G}=$ $0.25 \mathrm{~V}$ and $V_{D}=0.5 \mathrm{~V}$.

$\mathrm{eV}$ based on the scissors scheme [12], following the estimated range of $E_{g}=1.48-1.60 \mathrm{eV}$ in literature [8]. Note that this band gap adjustment does not affect our results at all as we explore only n-type transport behaviors of GeH FET in this study, where the exact value of $E_{g}$ is out of the picture.

To obtain the tight-binding (TB) parameters from the DFT band structure, two-center Slater-Koster approximation [13] was used with second-nearest neighbors. $s$ and $p$ atomic orbitals were considered for Ge, while only $s$ orbital is taken into account for $\mathrm{H}$. We used an orthonormal basis set. Mean squared error was employed to fit the TB band structure to the DFT one. The resulted TB parameters fit the band structure very accurately near the band edges as shown in Fig. 1(a).

Figure 1(b) shows the simulated device structure. Monolayer $\mathrm{GeH}$ was used for the channel, and source and drain are n-doped with a doping concentration of $5.5 \times 10^{-12} \mathrm{~cm}^{-2}$. A single-gate device structure is employed through $2.7-\mathrm{nm}$-thick $\mathrm{Al}_{2} \mathrm{O}_{3}(\kappa=9)$ for a gate dielectric. The nominal device has a 14-nm channel.

To assess the performance limit of GeH FETs, we have run atomistic quantum transport simulations using the non-equilibrium Green's function (NEGF) method within tight-binding approximations. Transport equations are solved iteratively with Poisson's equation until self-consistency between charge density and electrostatic potential is achieved [14]. Periodic boundary condition is applied for the width of device, and the charge and the current are calculated by taking the summation of transverse modes in the width direction. Ballistic transport is assumed since the channel length is very short and hence the effect of scattering is expected to be minimal. Open boundary conditions are treated with contact self-energies using the Neumann boundary condition. A power supply voltage $V_{D D}=0.5 \mathrm{~V}$ (which is smaller than the ITRS requirement) and room temperature are used.

\section{RESUltS}

Figure 2(a) shows transfer characteristics of 14-nm-channel monolayer GeH FET (solid lines) at $V_{D}=0.5 \mathrm{~V}$, plotted both in a logarithmic scale (left axis) and linear scale (right axis). It exhibits excellent switching characteristics with small subthreshold swing (SS $\sim 64 \quad \mathrm{mV} / \mathrm{dec}$ ) and a large maximum-achievable on/off ratio $\left(\sim 10^{12}\right)$ as well as high maximum-achievable on current $\left(I_{\text {on }}>2 \mathrm{~mA} / \mu \mathrm{m}\right.$; at $V_{\text {on }}=1.2 \mathrm{~V}$ and $\left.V_{\text {off }}=V_{o n}-V_{D D}\right)$. Note that this large $I_{o n}$ can be obtained at the sacrifice of $I_{o f f}$, and we will discuss the detailed relation
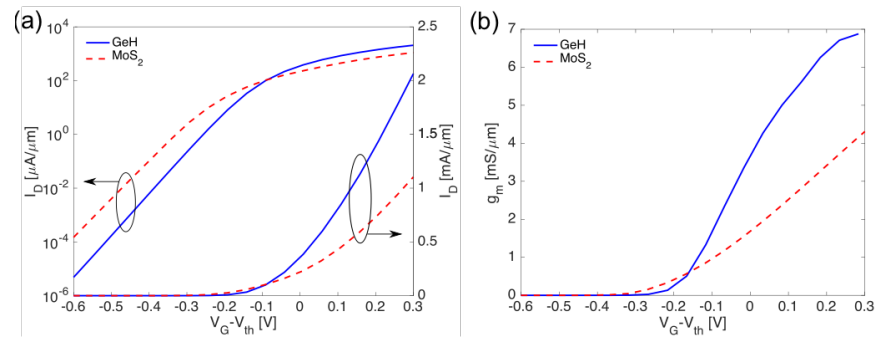

Fig. 3 (a) $I_{D}$ vs. $V_{G}-V_{t h}$ for GeH FET and $\mathrm{MoS}_{2}$ FET both with $L_{c h}=14 \mathrm{~nm}$ at $V_{D}=0.5 \mathrm{~V}$, considering threshold voltage shift. (b) Transconductance $g_{m}$ vs. $V_{G}-V_{t h}$ for the devices shown in (a).

between $I_{o n}$ and $I_{o n} / I_{\text {off }}$ in Fig. 4(d). It should be also noted that the current shown in this study is calculated without considering contact resistance to investigate the performance limit of GeH FETs. If the contact resistance is taken into account, current will be reduced to some extent due to the voltage drop across it and hence the lower effective gate and drain voltages across the device, depending on the actual quality of contacts. In spite of its excellent switching characteristic below the subthreshold voltage, the GeH FET eventually loses its steepness in subthreshold slope at lower gate voltages $\left(0<V_{G}<0.25 \mathrm{~V}\right.$ in Fig. 2(a)), unlike other 2D-material FETs. This can be understood by investigating the energy-resolved current spectrum as plotted in Fig. 2(b) for 14-nm-channel GeH FET at $V_{G}=0.25 \mathrm{~V}$. It reveals that the contribution of tunneling current $\left(I_{\text {tunneling }}\right)$ to the total current $\left(I_{\text {total }}=I_{\text {thermionic }}+I_{\text {tunneling }}\right)$ is prominent as thermionic current (Ithermionic $)$ becomes smaller at low gate voltages, resulting in the increase of subthreshold swing. Note that this conspicuous tunneling through 14-nm channel in GeH FET is attributed to its very light effective mass $\left(0.07 m_{0}\right)$, whereas $\mathrm{MoS}_{2}$ is known for its sustainability at much shorter channel lengths due to its heavier electron effective mass $\left(0.55 m_{0}\right)$ [15].

We also simulated 10-nm-channel GeH FET (dashed lines in Fig. 2(a)) and compared it against 14-nm-channel device. It turned out that the subthreshold swing of $10-\mathrm{nm}$-channel GeH FET is remarkably greater than $14-\mathrm{nm}$ device due to the significantly larger tunneling current through the thinner barrier. However, both devices having different channel lengths show almost same on current since $I_{o n}$ is dictated mainly by thermionic current at high gate voltages where the effect of tunneling current is infinitesimal.

It will be instructive to benchmark GeH FETs against other similar 2D semiconductor devices. Therefore, here we also simulate 14-nm-channel $\mathrm{MoS}_{2}$ FET within tight-binding approximation [16], and compare the device characteristics of GeH FET and $\mathrm{MoS}_{2}$ FET. Figures 3(a) and 3(b) are $I_{D}$ and $g_{m}(=$ $\left.\partial I_{D} / \partial V_{G}\right)$ as a function of $\left(V_{G}-V_{t h}\right)$, respectively. While both devices show nearly ideal switching characteristics ( $\mathrm{SS}=64$ $\mathrm{mV} / \mathrm{dec}$ for $\mathrm{GeH} ; 67.8 \mathrm{mV} / \mathrm{dec}$ for $\mathrm{MoS}_{2}$ ), GeH FET exhibits significantly better on-state characteristics with $2 \times$ larger $I_{\text {on }}$ and $g_{m}$ than $\mathrm{MoS}_{2}$ FET, which indicates that the gain in carrier velocity is greater than the loss in density of states (DOS) and quantum capacitance $\left(C_{Q}\right)$ through its small effective mass. In principle, higher- $\kappa$ or thinner dielectric can further boost $I_{o n}$ by increasing oxide capacitance $\left(C_{o x}\right)$ at the classical capacitance limit, particularly for the channel material with large $m^{*}$ like 


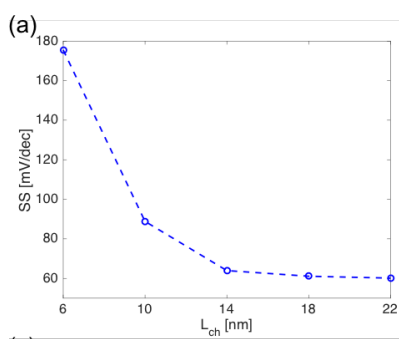

(c)
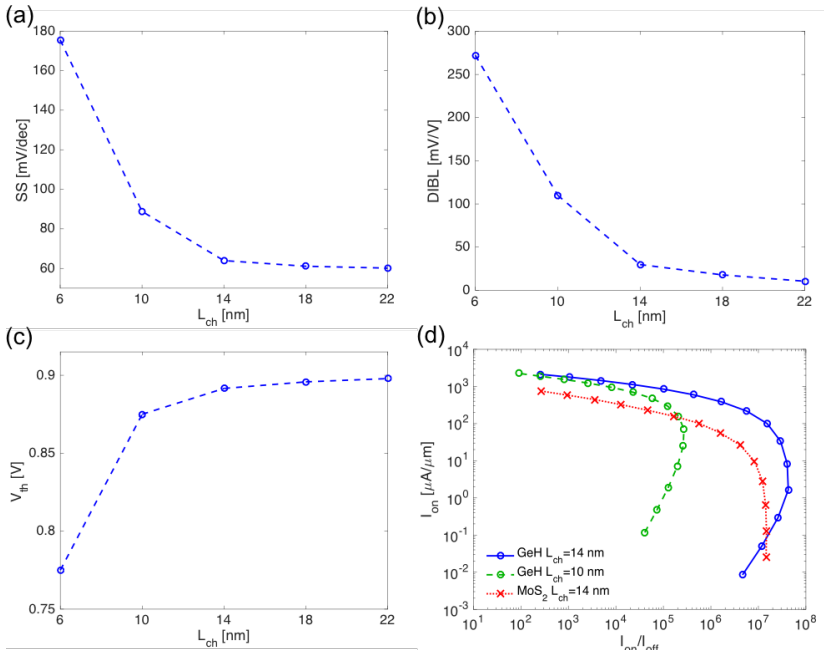

Fig. 4 (a) Subthreshold swing (SS), (b) drain-induced barrier lowering (DIBL), and (c) threshold voltage $\left(V_{t h}\right)$ as a function of channel length for the GeH FET. (d) $I_{o n}$ vs. $I_{o n} / I_{o f f}$ for GeH FETs (10-nm and $14-\mathrm{nm}$ channel) and a $\mathrm{MoS}_{2}$ FET (14-nm channel).

$\mathrm{MoS}_{2}$. However, for GeH FETs, such improvement can be limited due to extremely small $m^{*}$, low DOS and small $C_{Q}$. Nonetheless, in order to investigate the detailed effects of larger $C_{o x}$ on device performance, further study will be needed with full self-consistent simulations considering different equivalent oxide thickness since the total gate capacitance is a strong non-linear function of surface potential and charge, which is beyond the scope of this study.

In general, channel length $\left(L_{c h}\right)$ is one of the most important device parameters that determine the overall performance of FETs. Moreover, as we have seen in Fig. 2, device performance of GeH FET can be very susceptible to the actual channel length. Therefore, next, we perform a channel length scaling study of monolayer $\mathrm{GeH}$ FETs. Figure 4(a) shows SS (= $\left.\partial V_{G} / \partial \log _{10}\left(I_{D}\right)\right)$ for various channel lengths from 6 to $22 \mathrm{~nm}$. For the device with $L_{c h} \geq 14 \mathrm{~nm}, \mathrm{SS}$ is close to the theoretical limit of $60 \mathrm{mV} / \mathrm{dec}$, but it shows significant increases at sub-10-nm channel lengths, resulting in $175 \mathrm{mV} / \mathrm{dec}$ at $L_{c h}=6$ $\mathrm{nm}$. Similar trend can also be observed for drain-induced barrier lowering (DIBL $=\Delta V / \Delta V_{D}$ ) in Fig. 4(b), which is calculated at $I_{\text {off }}=10^{-3} \mu \mathrm{A} / \mu \mathrm{m}$ using $V_{D}=0.05 \mathrm{~V}$ and $0.5 \mathrm{~V}$. Thus, it can be concluded from Figs. 4(a) and 4(b) that monolayer GeH FET can be scaled down to $\sim 14 \mathrm{~nm}$ without facing significant short-channel effects. We have also investigated how threshold voltage is affected by channel length scaling in Fig. 4(c). The threshold voltage is $\sim 0.89 \mathrm{~V}$ for the channel longer than $14 \mathrm{~nm}$, but it shows $V_{t h}$ roll-off at sub-10-nm channel, resulting in $V_{t h}=0.775 \mathrm{~V}$ at $L_{c h}=6 \mathrm{~nm}$.

Finally, for comprehensive analyses, we plotted $I_{o n}$ vs. $I_{o n} / I_{\text {off }}$ for three different devices of GeH FETs (10-nm and 14-nm channel) and a $\mathrm{MoS}_{2}$ FET (14-nm channel) in Fig. 4(d), where we have observed the following points: (1) While both 10-nm and 14-nm-channel GeH FETs can equally provide as high on current as $2 \mathrm{~mA} / \mu \mathrm{m}, I_{\text {on }} / I_{\text {off }}$ is significantly larger with 14-nm-channel device for the same $I_{o n}$. For example, at a common $I_{o n}=100 \mu \mathrm{A} / \mu \mathrm{m}, I_{o n} / I_{\text {off }}=1.53 \times 10^{7}$ with $14-\mathrm{nm}$ channel, whereas $I_{\text {on }} / I_{\text {off }}$ of $10-\mathrm{nm}$ channel device is $2.42 \times 10^{5}$. (2) Although both GeH FET and $\mathrm{MoS}_{2}$ FET with the same 14-nm channel can provide as large on-off current ratio as $I_{\text {on }} / I_{\text {off }}>10^{7}, I_{\text {on }}$ of GeH FET can be significantly higher than that of $\mathrm{MoS}_{2}$ FET for the same $I_{\text {on }} / I_{\text {off. }}$. For instance, at a common $I_{\text {on }} / I_{\text {off }}=10^{5}$, on current is $850 \mu \mathrm{A} / \mu \mathrm{m}$ and $183 \mu \mathrm{A} / \mu \mathrm{m}$ for GeH FET and $\mathrm{MoS}_{2}$ FET, respectively. (3) In addition, we notice a unique shape of the curves in the $I_{o n}$ vs. $I_{o n} / I_{\text {off }}$ plots for GeH FETs, which can be distinguished from those of other 2D-material FETs such as $\mathrm{MoS}_{2}$ (dotted line with crosses in Fig. 4(d)) and black phosphorus [17]. While other materials show the monotonic increase of $I_{o n} / I_{\text {off }}$ by sacrificing $I_{o n}$, GeH FETs exhibit non-monotonic behaviors due to the tunneling current at low gate voltages as discussed earlier.

\section{CONCLUSION}

We explored the performance limit of monolayer GeH FETs using self-consistent atomistic quantum transport simulations. GeH FET exhibits superior on-state device performance such as high $I_{o n}(>2 \mathrm{~mA} / \mu \mathrm{m})$ and large $g_{m}(\sim 7 \mathrm{mS} / \mu \mathrm{m})$, due to its very light effective mass, as well as excellent switching characteristics ( $\mathrm{SS} \sim 64 \mathrm{mV} / \mathrm{dec}$ ). Our scaling study revealed that $\sim 14 \mathrm{~nm}$ will be suitable for the channel length of GeH FET as it may suffer from significant short channel effects if the channel length becomes less than $10 \mathrm{~nm}$. We have also benchmarked GeH FET against $\mathrm{MoS}_{2}$ device, which suggested that $\mathrm{GeH}$ has clear benefits for high-performance device applications over $\mathrm{MoS}_{2}$.

\section{REFERENCES}

[1] Y. Yoon, K. Ganapathi, and S. Salahuddin, "How good can monolayer $\mathrm{MoS}_{2}$ transistors be?," Nano Lett., vol. 11, no. 9, pp. 3768-73, 2011.

[2] D. Yin and Y. Yoon, "Design strategy of two-dimensional material field-effect transistors: Engineering the number of layers in phosphorene FETs," J. Appl. Phys., vol. 119, p. 214312, 2016.

[3] S. Bertolazzi, D. Krasnozhon, and A. Kis, "Nonvolatile Memory Cells Based on $\mathrm{MoS}_{2}$ /Graphene Heterostructures," ACS Nano, vol. 7, no. 4, pp. 3246-3252, 2013.

[4] F. Xia, H. Wang, and Y. Jia, "Rediscovering black phosphorus as an anisotropic layered material for optoelectronics and electronics," Nat. Commun., vol. 5, p. 4458, 2014.

[5] J. Kwon, Y. K. Hong, G. Han, I. Omkaram, W. Choi, S. Kim, and Y. Yoon, "Giant Photoamplification in Indirect-Bandgap Multilayer $\mathrm{MoS}_{2}$ Phototransistors with Local Bottom-Gate Structures," Adv. Mater., vol. 27, no. 13, pp. 2224-2230, 2015.

[6] Y. Wu, Y. Lin, A. A Bol, K. A Jenkins, F. Xia, D. B. Farmer, Y. Zhu, and P. Avouris, "High-frequency, scaled graphene transistors on diamond-like carbon.," Nature, vol. 472, no. 7341, pp. 74-78, 2011.

[7] V. E. Dorgan, M. H. Bae, and E. Pop, "Mobility and saturation velocity in graphene on $\mathrm{SiO}_{2}$," Appl. Phys. Lett., vol. 97, no. 8, pp. 8-11, 2010.

[8] E. Bianco, S. Butler, S. Jiang, O. D. Restrepo, W. Windl, and J. E. Goldberger, "Stability and Exfoliation of Germanane: A Germanium Graphane Analogue," ACS Nano, vol. 7, no. 5, pp. 4414-4421, 2013.

[9] R. K. Ghosh, M. Brahma, and S. Mahapatra, "Germanane: A low effective mass and high bandgap 2-D channel material for future FETs," IEEE Trans. Electron Devices, vol. 61, no. 7, pp. 23092315, 2014.

[10] K. L. Low, W. Huang, Y. C. Yeo, and G. Liang, "Ballistic transport performance of silicane and germanane transistors," IEEE Trans. Electron Devices, vol. 61, no. 5, pp. 1590-1598, 
2014.

[11] J. M. Soler, E. Artacho, J. D. Gale, A. Garcia, J. Junquera, P. Ordejon, and D. Sanchez-Portal, "The SIESTA method for ab initio order-N materials simulation," J. physics. Condens. matter, vol. 14, no. 11, pp. 2745-2779, 2002.

[12] C. W. M. Castleton, A. Höglund, and S. Mirbt, "Density functional theory calculations of defect energies using supercells," Model. Simul. Mater. Sci. Eng., vol. 17, p. 84003, 2009.

[13] J. C. Slater and G. F. Koster, "Simplified LCAO Method for the Periodic Potential Problem," Phys. Rev., vol. 94, no. 6, pp. 1498 1524, 1954.

[14] S. Datta, Quantum Transport: Atom to Transistor. Cambridge University Press, 2005.

[15] S. B. Desai, S. R. Madhvapathy, A. B. Sachid, J. P. Llinas, Q. Wang, G. H. Ahn, G. Pitner, M. J. Kim, J. Bokor, C. Hu, A. Javey, H.-S. P. Wong, and A. Javey, " $\mathrm{MoS}_{2}$ transistors with 1-nanometer gate lengths," Science, vol. 354, no. 6308, pp. 99-102, 2016.

[16] G. Bin Liu, W. Y. Shan, Y. Yao, W. Yao, and D. Xiao, "Three-band tight-binding model for monolayers of group-VIB transition metal dichalcogenides," Phys. Rev. B - Condens. Matter Mater. Phys., vol. 88, no. 8, p. 085433, 2013.

[17] D. Yin, G. Han, and Y. Yoon, "Scaling limit of bilayer phosphorene FETs," IEEE Electron Device Lett., vol. 36, no. 9, pp. 978-980, 2015. 